\title{
Elemental Analysis of Rat's Femoral Neck with Experimental Diabetes by means of Scanning Electron Microscopy
}

\author{
Gabriel Herrera-Pérez ${ }^{1 \mathbf{a}}$, Xóchitl Sofía Ramírez-Gómez ${ }^{2 a}$, Rafael Vargas-Bernal ${ }^{1}$, Esmeralda Rodríguez- \\ Miranda ${ }^{3}$. \\ ${ }^{1}$ Departamento de Ingeniería en Materiales, Instituto Tecnológico Superior de Irapuato, México. \\ ${ }^{2}$ Departamento de Enfermería Clínica, División de Ciencias de la Salud e Ingeniería, Universidad de \\ Guanajuato Campus Celaya, México. \\ ${ }^{3}$ Departamento de Medicina y Nutrición, División de Ciencias de la Salud, Universidad de Guanajuato \\ Campus León, México.
}

Diabetes mellitus (DM) is a group of metabolic disorders, whose main characteristic is the hyperglycemia. The duration of the hyperglycemia as well as its severity, are the most important factors that establish its presence by intermediate or prolonged periods. Although, pathophysiological changes that accompany the DM are found in all body, there are specific clinical manifestations such as gradual vision loss, renal disease, and susceptibility to cardiovascular diseases. In addition, the skeleton is affected to a significant level, where a major complication is osteoporotic bone fracture. Both patients with type 1 (T1DM) and type 2 (T2DM) diabetes mellitus have a high risk to experience fracture (Vestergaard et al 2009), when its condition is compared to health individuals. Reported data suggest that patients with T1DM show a decrease in bone mineral density (BMD), which is attributed to the decrease of the bone formation during growth in children and adolescents (Hamann et al 2012). In contrast, adults with T2DM, BMD values are normal or slightly elevated; however, the risk of a fracture is high (Janghorbani et al 2007). This advises that these patients may have a poor bone quality, which cannot be estimated in conventional densitometry studies. In this paper, we use a model of experimental diabetes (ED) to evaluate macroscopically the femur; representing the part of the skeleton, in which fractures caused by the weaking of the skeleton occur more frequently.

In this study, female Wistar rats were used, with 8 to 12 weeks old, and weighing 200 to $250 \mathrm{~g}$. The slaughter and handling of animals was made considering the specifications outlined in the Official Mexican Standard NOM-062-ZOO-1999. Before the induction of ED, the rats were fasted for 4 hours, after this time, alloxan monohydrate (Sigma-Aldrich) was administrated intraperitoneally $(180 \mathrm{mg} / \mathrm{kg}$ ). Animals with blood glucose values $>150 \mathrm{mg} / \mathrm{dL}$ were considered diabetic. Rats were maintained under these conditions for 12 months before sacrifice. Prior to sacrifice, body weight and blood glucose level were recorded. The rats were sacrificed by cervical dislocation and immediately skeleton dissection was performed. To prevent damage to the bone by a chemical treatment, the bones were cleaned using beetles, Coleoptera order and Dermestidae family.

Our results show that the glucose level increased significantly from $53 \pm 8$ in the group for non-diabetic rats to values of $* 234 \pm 39$ for the group of diabetic. ${ }^{*} p<0.05$ student's t-test, $\mathrm{n}=3$ , two weeks after induction and kept under these conditions for 12 months. This glucose level in rats corresponds to the clinical condition of non-controlled diabetic patients. Femur macroscopic parameters such as weight, length, and diameter of the diaphysis decreased $\sim 40 \%, \sim 10 \%, \sim 7 \%$ respectively; while the diameter of the upper and lower epiphysis increased $\sim 2 \%, \sim 10 \%$. Analysis by Scanning Electron Microscopy (SEM) shows that the group of rats with ED increased femoral neck diameter 30\% resulting in widening and deformation of the head of the bone (see Figure 1A and 1B).

a Authors with the contribution of first author. 


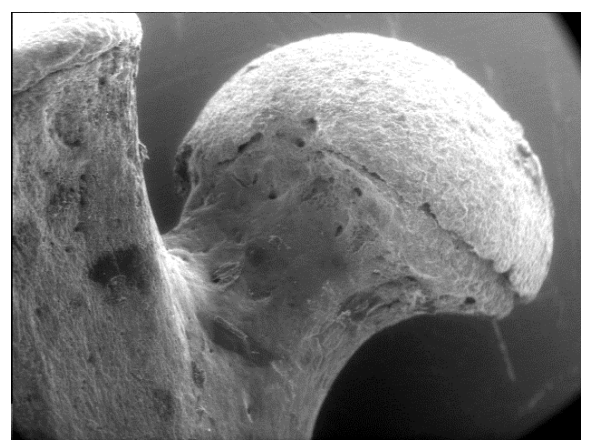

(A)

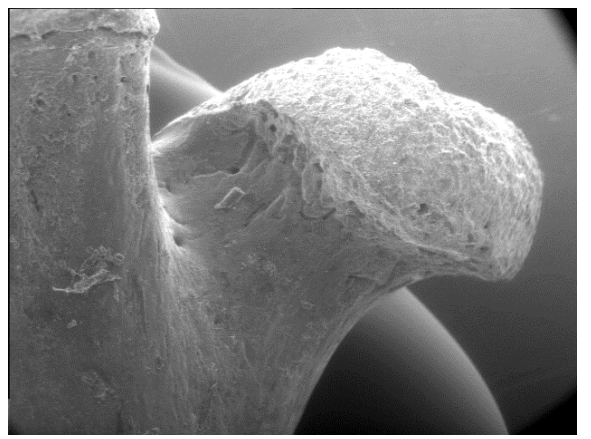

(B)

Figure 1. Images of SEM for Non-Diabetic (A) and Diabetic (B) Rats 25x, Electron Source Gun, 15.0 KV, Spot Magnitude 4.0, Pressure 0.8 Torr.

Clinical studies have shown that the femoral neck is the site in the body, where fractures caused by osteoporosis occur more frequently (Kosy et al 2013). Elemental analysis of samples by Energy Dispersive Spectroscopy (EDS-SEM) provides that the content of $\mathrm{K}$ is similar in both cases $\sim 0.40 \%$ (At-g). The content of $\mathrm{Ca}, \mathrm{Na}, \mathrm{P}$, and $\mathrm{O}$ decreased in the diabetic group (Table 1), while for the case of $\mathrm{N}$ y Mg had an increase, all these differences were statistically significant ( ${ }^{*} p<0.05$ student's t-test).

Table 1. Elemental analysis of the surface of the femoral neck.

\begin{tabular}{lcccccccccc}
\hline \multirow{2}{*}{ Group } & \multicolumn{10}{c}{ Elemental Analysis EDS (\% At-g) } \\
\cline { 2 - 10 } & $\mathbf{C}$ & $\mathbf{N}$ & $\mathbf{O}$ & $\mathbf{N a}$ & $\mathbf{M g}$ & $\mathbf{C a}$ & $\mathbf{P}$ & Others & $\mathbf{C a} / \mathbf{P}$ \\
\hline \multirow{2}{*}{ Non-Diabetic } & 31.99 & 6.87 & 44.38 & 0.43 & 0.18 & 9.19 & 4.95 & 2.13 & \multirow{2}{*}{1.86} \\
& \pm 1.15 & \pm 0.18 & \pm 0.59 & \pm 0.05 & \pm 0.03 & \pm 0.65 & \pm 0.40 & N/A & \\
Diabetic & $37.48^{*}$ & $7.76^{*}$ & $41.21^{*}$ & $0.33^{*}$ & $0.21^{*}$ & $6.32^{*}$ & $3.90^{*}$ & 0.80 & \multirow{2}{*}{1.62} \\
& \pm 3.18 & \pm 0.40 & \pm 2.84 & \pm 0.05 & \pm 0.02 & \pm 1.18 & \pm 0.16 & N/A & \\
\hline
\end{tabular}

The stoichiometric ratio $\mathrm{Ca} / \mathrm{P}$ in the hydroxiapatite (HA) is 1.667 , however, it has been reported that an optimum value of this ratio in the femur of healty female Wistar rat is 1.664, and $\sim 2.1$ if they are rats with 8 to 12 weeks of age (Hernández-Urbiola et. al. 2012). In this paper, the ratio of $\mathrm{Ca} / \mathrm{P}$ of 1.856 was found in the HA for the group of non-diabetic rats and a value of 1.622 for the diabetic rats, both groups had 48 weeks of age when elemental analysis was performed. Although, the onset and development of osteoporosis is commonly asymptomatic; by reducing the weight, length and diameter of the diaphysis of the bone, does conclude that the experimental group with DM also developed secondary osteoporosis, that is, an effect caused by some other pathology and/or lifestyle and not due to age.

Acknowledgement: This project was supported by CONCYTEG for $7^{\text {th }}$ State Research Summer 2014.

Hamann C, Kirschner S, Günther KP, Hofbauer LC. 2012; 8(5): 297-305.

Hernández-Urbina M, Mineral Content and Physicochemical Properties in Female Rats Bone During Growing Stage, Absorption Atomic Spectroscopy 2012; 11: 201-220.

Janghorbani M, Van Dam RM, Willett WC, Hu FB. Am J Epidemiol 2007; 166(5): 495-505.

Kosy JD, Blackshaw R, Swart M, Fordyce A, Lofthouse R.A. J. Orthop Traumatol. 2013 Sep; 14(3):165-70.

NORMA Oficial Mexicana NOM-062-ZOO-1999, Especificaciones técnicas para producción, cuidado y uso de los animales de laboratorio. http://www2.inecc.gob.mx/publicaciones/gacetas/220/062.html Vestergaard P, Rejnmark L, Mosekilde L. Calcif Tissue Int 2009; 84(1): 45-55. 